\title{
Ozsváth-Szabó and Rasmussen invariants of doubled knots
}

\author{
Charles Livingston \\ SWATEE NAIK
}

Let $v$ be any integer-valued additive knot invariant that bounds the smooth 4-genus of a knot $K,|v(K)| \leq g_{4}(K)$, and determines the 4-ball genus of positive torus knots, $v\left(T_{p, q}\right)=(p-1)(q-1) / 2$. Either of the knot concordance invariants of Ozsváth-Szabó or Rasmussen, suitably normalized, have these properties. Let $D_{ \pm}(K, t)$ denote the positive or negative $t$-twisted double of $K$. We prove that if $v\left(D_{+}(K, t)\right)= \pm 1$, then $v\left(D_{-}(K, t)\right)=0$. It is also shown that $v\left(D_{+}(K, t)\right)=1$ for all $t \leq \mathrm{TB}(K)$ and $v\left(D_{+}(K, t)\right)=0$ for all $t \geq-\mathrm{TB}(-K)$, where $\operatorname{TB}(K)$ denotes the Thurston-Bennequin number.

A realization result is also presented: for any $2 g \times 2 g$ Seifert matrix $A$ and integer $a,|a| \leq g$, there is a knot with Seifert form $A$ and $v(K)=a$.

57M27; 57M25

\section{Introduction}

Two recently discovered smooth knot concordance invariants, the Ozsváth-Szabó invariant $\tau$, [8], and the Rasmussen invariant $s$, [10], have opened up powerful new perspectives on the study of concordance. For instance, each is sufficient to prove the Milnor conjecture determining the smooth 4-ball genus of torus knots, and each demonstrates the existence of non-slice Alexander polynomial one knots. Unfortunately, the computation of $\tau$ is not algorithmic and $s$, though algorithmic, is difficult to compute for classes of knots. The only classes for which general results are known are alternating knots and torus knots (Ozsváth-Szabó [8], Rasmussen [10]), and quasipositive knots (Livingston [5], Shumakovich [12]). Related results are included in Eftekhary, HeddenOrding, Owens-Strle and Plamenevskaya $[3 ; 4 ; 7 ; 9]$. Of special relevance here are the recent results for doubles of $(2, n)$-torus knots by Matt Hedden and Philip Ording [4], discussed further in an addendum to this paper.

The three observations of this note grew out of efforts to compute $\tau$ and $s$. In [5], methods for computing $\tau$ were developed and used to prove that some untwisted doubles 
of knots have non-vanishing $\tau$. The results of [5] depended on only three properties of $\tau$ : (1) $\tau(K \# J)=\tau(K)+\tau(J)$, (2) $|\tau(K)| \leq g_{4}(K)$, and (3) $\tau\left(T_{p, q}\right)=(p-1)(q-1) / 2$ for all torus knots $T_{p, q}$ with $p, q>0$. The invariant $s$ shares these properties of $\tau$ when suitably normalized; more precisely, $-s / 2$ has these three properties. Thus, for this paper we refer to an arbitrary knot invariant having these three properties as $v$.

Let $D_{ \pm}(K, t)$ denote the positive or negative $t$-twisted double of $K$. Since $D_{ \pm}(K, t)$ bounds a surface of genus $1, \mid v\left(D_{ \pm}(K, t) \mid \leq 1\right.$. Our results are the following.

Theorem 1 If $v\left(D_{+}(K, t)\right)= \pm 1$, then $v\left(D_{-}(K, t)\right)=0$.

Associated to a knot $K$ there is an integer-valued invariant called the ThurstonBennequin number, $\mathrm{TB}(K)$, first defined in Bennequin [2]. This invariant was initially defined in terms of Legendrian structures, but there is the following combinatorial definition. Every knot has some diagram so that in a neighborhood of each crossing the projection consists of two segments, one with slope 1 and the other of slope -1 . Furthermore it can be arranged that the segment with slope 1 passes under the other segment. For such a diagram, the Thurston-Bennequin number is the writhe of the diagram minus the number of right cusps (maximum points with respect to projection onto the $x$-axis). The Thurston-Bennequin number of the knot is the maximum value of this difference, taken over all diagrams satisfying the crossing criteria.

For further details about the Thurston-Bennequin number, see $\mathrm{Ng}$ [6]. Note that $\mathrm{TB}(-K) \neq-\mathrm{TB}(K)$; in fact, for all $K, \mathrm{~TB}(-K)+\mathrm{TB}(K) \leq-1$.

Theorem 2 For each knot $K$ there is an integer $t_{K}$ such that $v\left(D_{+}(K, t)\right)=1$ for $t \leq t_{K}$ and $v\left(D_{+}(K, t)\right)=0$ for all $t>t_{K}$. The value of $t_{K}$ satisfies $T B(K) \leq t_{K}<$ $-T B(-K)$

Similar results hold with the roles of $D_{+}$and $D_{-}$reversed.

Techniques related to those we use here to study doubled knots also quickly yield the following.

Theorem 3 For any $2 g \times 2 g$ Seifert matrix $A$ and integer $a,|a| \leq g$, there is a knot $K$ bounding a genus $g$ Seifert surface with Seifert form $A$ and $\nu(K)=a$.

Acknowledgments We thank Matt Heddon for sharing the results of his work with Philip Ording with us. We also thank Ciprian Manolescu and Brendan Owens for identifying an error in sign conventions in an early version of this paper.

Algebraic 83 Geometric Topology, Volume 6 (2006) 


\section{Band modifications and 4-genus}

A Seifert surface for a knot, viewed as a disk with bands attached, can be modified by removing one of the bands and reattaching it in the same place, perhaps twisted and knotted in a different way. We call this operation band modification. For example, the first diagram in Figure 1 illustrates the knot $D_{+}(K, t)$ : the band on the right has framing $t$. A band modification is performed on the left band to yield $D_{-}(K, t)$. A band modification is then performed on the right band to yield $D_{-}(-K,-t)$, and we note that $D_{-}(-K,-t)=-D_{+}(K, t)$.
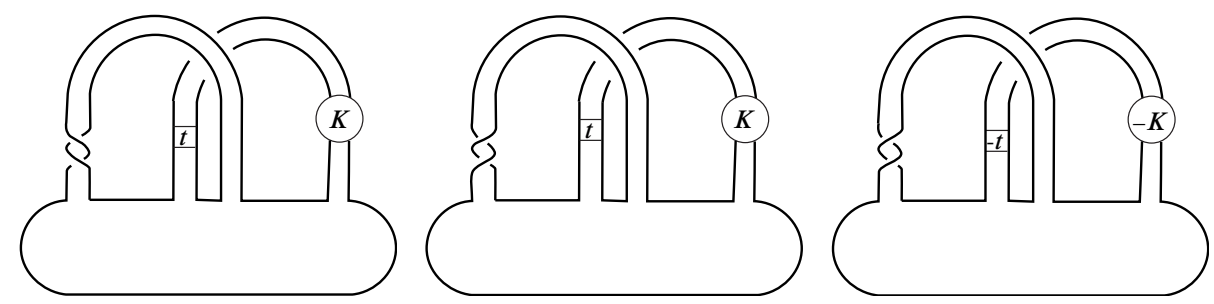

Figure 1: $\quad D_{+}(K, t), \quad D_{-}(K, t)$, and $D_{-}(-K,-t)=-D_{+}(K, t)$

Theorem 4 If a knot $K^{\prime}$ is constructed from a knot $K$ by performing a single band modification, then $K \#-K^{\prime}$ has smooth 4-genus 0 or 1.

Proof For any knot $J$, removing a single band in the Seifert surface yields a 2component link $L$, and there is a genus 0 cobordism from $J$ to $L$. In our setting removing the corresponding bands from $K$ and $K^{\prime}$ yields the same link $L$. Gluing the two cobordisms together along $L$ yields a genus 1 cobordism from $K$ to $K^{\prime}$. The standard construction of removing the neighborhood of an arc from $K$ to $K^{\prime}$ on that cobordism yields a punctured surface of genus 1 , bounded by $K \#-K^{\prime}$, embedded in $B^{4}$.

We assume now and for the rest of the paper that $v$ is an integer-valued additive knot invariant satisfying $|v(K)| \leq g_{4}(K)$ for all $K$ and $v\left(T_{p, q}\right)=(p-1)(q-1) / 2$ for all torus knots $T_{p, q}$ with $p, q>0$.

Corollary 5 If $K^{\prime}$ is constructed from $K$ by a band modification, then $\mid v(K)-$ $v\left(K^{\prime}\right) \mid \leq 1$.

Proof Since $K^{\prime} \#-K^{\prime}$ is slice, $\left|v\left(K^{\prime}\right)+v\left(-K^{\prime}\right)\right|=\left|v\left(K^{\prime} \#-K^{\prime}\right)\right|=0$ and so $v\left(-K^{\prime}\right)=-v\left(K^{\prime}\right)$. By Theorem 4 we then have $\left|v(K)-v\left(K^{\prime}\right)\right|=\left|v\left(K \#-K^{\prime}\right)\right| \leq 1$. 


\section{Knot invariants and doubles}

Theorem 1 If $v\left(D_{+}(K, t)\right)= \pm 1$ then $v\left(D_{-}(K, t)\right)=0$.

Proof We have that $v\left(-D_{+}(K, t)\right)=\mp 1$. From the example illustrated in Figure 1, we see that $D_{-}(K, t)$ can be constructed from $D_{+}(K, t)$ and also from $-D_{+}(K, t)$ by a single band modification. Thus, by Corollary $5, v\left(D_{-}(K, t)\right)$ differs from both 1 and -1 by at most 1 , so it must be 0 .

Theorem 2 For each knot $K$ there is an integer $t_{K}$ such that $v\left(D_{+}(K, t)\right)=1$ for $t \leq t_{K}$ and $v\left(D_{+}(K, t)\right)=0$ for $t>t_{K}$. The value of $t_{K}$ satisfies $T B(K) \leq t_{K}<$ $-T B(-K)$.

Proof The construction here is much the same as one originated by Rudolph [11] as formulated in [5]; we only summarize it here.

For any $t \leq \mathrm{TB}(K), K$ can be isotoped to be on a minimal genus Seifert surface for some positive torus knot, $T_{p, q}$, with self-framing $t$. It follows that a genus one Seifert surface for $D_{+}(K, t)$ embeds on a torus knot Seifert surface. By results of [5] one then has $v\left(D_{+}(K, t)\right)=1$.

Similarly, for $t \leq \mathrm{TB}(-K), v\left(D_{+}(-K, t)\right)=1$. By Theorem 1, it follows that $v\left(D_{-}(-K, t)\right)=0$. Taking the mirror image, $v\left(D_{+}(K,-t)\right)=0$. Equivalently, $v\left(D_{+}(K, t)\right)=0$ if $t \geq-\mathrm{TB}(-K)$.

As proved in [5], the three conditions satisfied by $v$ imply that changing a positive crossing to a negative crossing in a knot diagram cannot increase the value of $v$ (and can decrease it by at most 1$)$. The knot $D_{+}(K, t)$ results from $D_{+}(K, t+1)$ when a negative crossing in the knotted band is changed into a positive crossing; notice that the crossings in a positively twisted band are negative crossings, since the strands are oriented in opposite directions. Thus, $v\left(D_{+}(K, t)\right)$ is a non-increasing function of $t$. The integer $t_{K}$ is defined to be the largest integer for which $v\left(D_{+}(K, t)\right)=1$. Its bounds follow from the results of the previous two paragraphs.

Example For the right-handed trefoil we have $\operatorname{TB}\left(T_{2,3}\right)=1$ and $\operatorname{TB}\left(-T_{2,3}\right)=-6$ (see [6]). Thus, $v\left(D_{+}\left(T_{2,3}, t\right)\right)=1$ for $t \leq 1$ and $v\left(D_{+}\left(T_{2,3}, t\right)\right)=0$ for $t \geq 6$. (See the addendum for remarks on recent work of Matt Hedden and Philip Ording [4] concerning the values of $\tau$ and $s$ for twisted doubles of $(2, n)$-torus knots.)

Remark The asymptotic limiting behavior of $v$ for doubled knots holds for more general families of companions. Suppose that $J$ is a winding number 0 knot in the 
solid torus that meets a meridinal disk transversely in exactly two points. Given any knot $K$, one can form the $t$-twisted satellite, $J(K, t)$, using $J$ as the companion. As $t$ is increased, $J(K, t)$ is changed by adding negative crossings. Thus, the value of $v(J(K, t))$ is non-increasing by a result of [5]. On the other hand, the genus of $J(K, t)$ is bounded above by the genus of a surface bounded by $J$ in the solid torus, so $v(J(K, t))$ is bounded below by the negative of that genus. It follows that the value of $v(J(K, t))$ has some finite limit as $t$ increases.

In the case that $J$ intersects the meridinal disk in more than 2 points, this argument does not work. Further properties of $\tau$ as it relates to surfaces in $C P^{2}$, proved in [8], can be applied to recover the asymptotic behavior in this case, but no similar argument is known for $s$.

\section{Realization result}

We now restate and prove the realization result:

Theorem 3 Given a $2 g \times 2 g$ Seifert matrix $A$, there are knots $K_{i},-g \leq i \leq g$, bounding Seifert surfaces of genus $g$ each with $A$ as its associated Seifert matrix, such that $v\left(K_{i}\right)=i$.

Proof It is essentially an observation of Rudolph, the Trefoil Insertion Lemma in [11], that by repeatedly adding trefoils to the bands of the Seifert surface in such a way that the framings of the bands, and thus the Seifert form, is unchanged, one eventually arrives at a surface $S$ that is isotopic to an embedded surface on a minimal genus Seifert surface for some positive torus knot. According to [5], for $K=\partial S$,

$$
v(K)=g_{4}(K)=g_{3}(K)=g(S) .
$$

Apply this construction for the Seifert matrix $A$ to build a knot $K_{+}$that bounds a genus $g$ Seifert surface $S_{+}$having Seifert form $A$ and $v\left(K_{+}\right)=g$. Similarly, build a knot $K_{-}$with genus $g$ Seifert surface $S_{-}$, Seifert form $-A$ and $v\left(K_{-}\right)=g$. Then $K_{+}$and $-K_{-}$both bound genus $g$ Seifert surfaces ( $S_{+}$and $-S_{-}$, respectively) with Seifert form $A$, where $v\left(K_{+}\right)=g$ and $v\left(-K_{-}\right)=-g$.

A series of $2 g$ band modification converts $S_{+}$into $-S_{-}$: just replace the bands for $S_{+}$with those of $-S_{-}$one at a time, maintaining the Seifert form at each step, as described in the following paragraph. Since each of the modifications changes the value of $v$ by at most 1 , and the $2 g$ modifications decrease $v$ by $2 g$, it follows that 
each step reduces $v$ by exactly one. Thus the sequence of knots arising from the series of modifications yields the desired examples.

To conclude the proof, we indicate the process of trading bands from those of the first surface to those of the second while maintaining the Seifert form. Given knots $K$ and $K^{\prime}$ with the same Seifert form, we can assume both are built from the same disk $D$ by adding bands, $\left\{b_{1}, \ldots b_{2 g}\right\}$ and $\left\{b_{1}^{\prime}, \ldots b_{2 g}^{\prime}\right\}$. We can further assume that the attaching maps for $b_{i}$ and $b_{i}^{\prime}$ are the same, and that all the bands are disjoint. The band $b_{1}$ can be removed and replaced with $b_{1}^{\prime}$. However, the linking numbers associated to $b_{1}^{\prime}$ with the other bands can differ from that of $b_{1}$. This is corrected by stretching $b_{1}^{\prime}$ to add clasps between it and the $b_{i}, i \geq 2$. Using an isotopy, $b_{1}^{\prime}$ can be returned to its original position, at the expense of moving the $b_{i}, i \geq 2$. This procedure can now be repeated, with $b_{2}$ and $b_{2}^{\prime}$, and so on, until the desired result is achieved.

Addendum (March, 2006) Since this paper was circulated, Hedden and Ording [4] have proved that the Ozsváth-Szabó invariants and the Rasmussen invariant are distinct. Let $t_{\tau}(K)$ and $t_{s}(K)$ denote the greatest value of $t$ for which $\tau\left(D_{+}(K, t)\right)=1$ or $s\left(D_{+}(K, t)\right)=-2$, a value which exists by the results of this paper. Hedden and Ording compute $t_{\tau}\left(T_{2,3}\right)=1$ but $t_{s}\left(T_{2,3}\right) \geq 2$. More generally, they show that $t_{\tau}\left(T_{2,2 n+1}\right)=2 n-1$ and computations of Rasmussen (usng Bar-Natan's program [1]) show that $t_{s}\left(T_{2,5}\right) \geq 5$, and $t_{s}\left(T_{2,7}\right) \geq 8$.

\section{References}

[1] D Bar-Natan, The Knot Atlas, http://www . math.toronto.edu/drorbn/KAtlas/

[2] D Bennequin, Entrelacements et équations de Pfaff, from: "Third Schnepfenried geometry conference, Vol. 1 (Schnepfenried, 1982)”, Astérisque 107, Soc. Math. France, Paris (1983) 87-161 MR753131

[3] E Eftekhary, Longitude Floer homology and the Whitehead double arXiv: math.GT/0407211

[4] M Hedden, P Ording, The Ozsváth-Szabó and Rasmussen concordance invariants are not equal arXiv:math.GT/0512348

[5] C Livingston, Computations of the Ozsváth-Szabó knot concordance invariant, Geom. Topol. 8 (2004) 735-742 MR2057779

[6] L L Ng, Maximal Thurston-Bennequin number of two-bridge links, Algebr. Geom. Topol. 1 (2001) 427-434 MR1852765

[7] B Owens, S Strle, The Ozsváth-Szabó and Rasmussen concordance invariants are not equal arXiv:math.GT/0312266 
[8] P Ozsváth, Z Szabó, Knot Floer homology and the four-ball genus, Geom. Topol. 7 (2003) 615-639 MR2026543

[9] O Plamenevskaya, Bounds for the Thurston-Bennequin number from Floer homology, Algebr. Geom. Topol. 4 (2004) 399-406 MR2077671

[10] J A Rasmussen, Khovanov homology and the slice genus arXiv:math.GT/0306378

[11] L Rudolph, Constructions of quasipositive knots and links. I, from: "Knots, braids and singularities (Plans-sur-Bex, 1982)", Monogr. Enseign. Math. 31, Enseignement Math., Geneva (1983) 233-245 MR728589

[12] A Shumakovitch, Rasmussen invariant, Slice-Bennequin inequality, and sliceness of knots arXiv:math.GT/0411643

Department of Mathematics, Indiana University, Bloomington, IN 47405, USA

Department of Mathematics and Statistics, University of Nevada, Reno, NV 89557, USA

livingst@indiana.edu, naik@unr.edu

Received: 26 February 2006

Algebraic $6 \mathcal{G}$ Geometric $\mathcal{T}$ opology, Volume 6 (2006) 